\title{
THE EVOLUTION OF AUTONOMIC SPACE AS A METHOD OF MENTAL WORKLOAD ASSESSMENT FOR DRIVING
}

\author{
John K. Lenneman \\ General Motors Research \& Development and Planning, \\ Warren, Michigan, USA \\ E-mail: john.lenneman@gm.com \\ Richard W. Backs \\ Central Michigan University \\ Mount Pleasant, Michigan, USA
}

\begin{abstract}
Summary: Because heart rate lacks diagnosticity, an autonomic space approach for the assessment of mental workload has been proposed. In addition to increasing the capability to identify differences between tasks, the autonomic space approach can be used to make better inferences about the psychological processes involved. In this paper, the approach and its application to a simulated driving task are discussed, as well as suggestions for future research and its development.
\end{abstract}

\section{INTRODUCTION}

Heart rate has probably been the most-used cardiovascular measure of mental workload in the field. However, while heart rate can provide information about the amount of workload imposed by a task, it often lacks sensitivity. For example, heart rate has not been found to differ between day and night conditions of flight, as the angle of approach increases, or between segments of flight simulation which contain extraordinary conditions such as engine failure as opposed to segments of flight such as cruise (Lewis, Jones, Austin \& Roman, 1967; Roscoe, 1978; Backs, Lenneman, \& Sicard, 1999).

Further, heart rate is not capable of providing diagnostic information regarding the source of mental workload. O'Donnell and Eggemeier (1986) define diagnosticity as the "capability of a technique to discriminate the amount of workload imposed in different operator capacities or resources" (p. 42-3). Backs (1995) identified two reasons for heart rate's limited diagnosticity. First, heart rate is affected by sources other than autonomic input that may be independent of mental workload. Second, heart rate alone provides limited information about the activity of the separate branches of the autonomic nervous system. Similar heart rate changes may be the result of different patterns of activity of the underlying autonomic nervous system that controls heart rate.

\section{THE MODES OF AUTONOMIC CONTROL}

The heart is dually-innervated by the sympathetic and parasympathetic branches of the autonomic nervous system. Sympathetic activation causes an increase in heart rate, whereas parasympathetic activation causes a decrease in heart rate. Traditionally, activity of these branches was thought to be reciprocal: sympathetic activation was coupled with parasympathetic withdrawal (increased heart rate), and parasympathetic activation was coupled with sympathetic 
withdrawal (decreased heart rate; Cannon, 1939). Any change in heart rate was always believed to be the result of some change in the reciprocity of the two branches (i.e., one of two modes of autonomic control).

However, the development of the "doctrine of autonomic space" (Berntson, Cacioppo, \& Quigley, 1991) suggests that the sympathetic and parasympathetic branches are not simply reciprocally coupled. Rather, their activity can be non-reciprocally coupled (coactivation or coinhibition) or even uncoupled (change in activity of one branch can occur independent of activity of the other). Thus, instead of a change in heart rate being the sum of activation of one branch and withdrawal of the other, eight modes of autonomic control exist (see Table 1).

\section{Table 1. The Eight Modes of Autonomic Control for Heart Rate}

\begin{tabular}{|c|c|c|c|c|}
\hline & & \multicolumn{3}{|c|}{ Parasympathetic Activity } \\
\hline & & $\begin{array}{c}\text { Parasympathetic } \\
\text { Activation }\end{array}$ & No Change & $\begin{array}{c}\text { Parasympathetic } \\
\text { Withdrawal }\end{array}$ \\
\hline \multirow{3}{*}{$\begin{array}{c}\text { Sympathetic } \\
\text { Activity }\end{array}$} & $\begin{array}{c}\text { Sympathetic } \\
\text { Activation }\end{array}$ & Coactivation & $\begin{array}{c}\text { Uncoupled } \\
\text { Sympathetic } \\
\text { Activation }\end{array}$ & $\begin{array}{c}\text { Reciprocally } \\
\text { Coupled }\end{array}$ \\
\hline & No Change & $\begin{array}{c}\text { Uncoupled } \\
\text { Parasympathetic } \\
\text { Activation } \\
\end{array}$ & No Change & $\begin{array}{c}\text { Uncoupled } \\
\text { Parasympathetic } \\
\text { Withdrawal }\end{array}$ \\
\hline & $\begin{array}{l}\text { Sympathetic } \\
\text { Withdrawal }\end{array}$ & $\begin{array}{c}\text { Reciprocally } \\
\text { Coupled }\end{array}$ & $\begin{array}{l}\text { Uncoupled } \\
\text { Sympathetic } \\
\text { Withdrawal }\end{array}$ & Coinhibition \\
\hline
\end{tabular}

The ability to decipher autonomic control modes provides the capability to detect differences between tasks that may not have been detected with heart rate alone (Backs, 1995). Yet, for the purposes of mental workload assessment, merely identifying differences between tasks is insufficient. As researchers we often would like to identify the sources for these differences. In order to do this, additional steps are needed to infer the psychological processes involved during the performance of a task.

\section{DECIPHERING PSYCHOLOGICAL-PHYSIOLOGICAL MAPPINGS}

Cacioppo and Tassinary (1990) provided a thorough review of how physiological measures can be used to infer the psychological processes involved during the performance of a task. Their primary contribution was that in order to develop a higher level of diagnosticity, there must exist a relation between the psychological processes and physiological measures termed a "marker." A marker relation is a one-to-one mapping of psychological processes and physiological measures. In a marker relation, a single physiological measure is sufficient to make inferences about the psychological process that produced it.

However, many psychological-physiological relations fall into the category Cacioppo and Tassinary (1990) termed an "outcome." An outcome relation is a many-to-one mapping of psychological processes to physiological responses. In this case, the level of diagnosticity significantly decreases because changes in a particular physiological response could be the result of a number of psychological processes, making inferences about the psychological processes 
involved in a particular task nearly impossible. In order to make the inferences we want, an outcome relation (a many-to-one relation) must be converted to a marker relation (a one-to-one relation; Cacioppo and Tassinary, 1990).

One method of converting an outcome relation to a marker relation is to redefine the elements in the physiological and/or psychological domains to create higher-order domains. Backs (2001) proposed that the element of heart rate be redefined as the modes of autonomic control. For example, tasks including working memory load, divided attention, and changes in tracking disturbance have all elicited increases in heart rate (Backs, 1995). Further analysis of the data by redefining heart rate as the autonomic control modes revealed that all three tasks elicited different modes of autonomic control (see Figure 1).

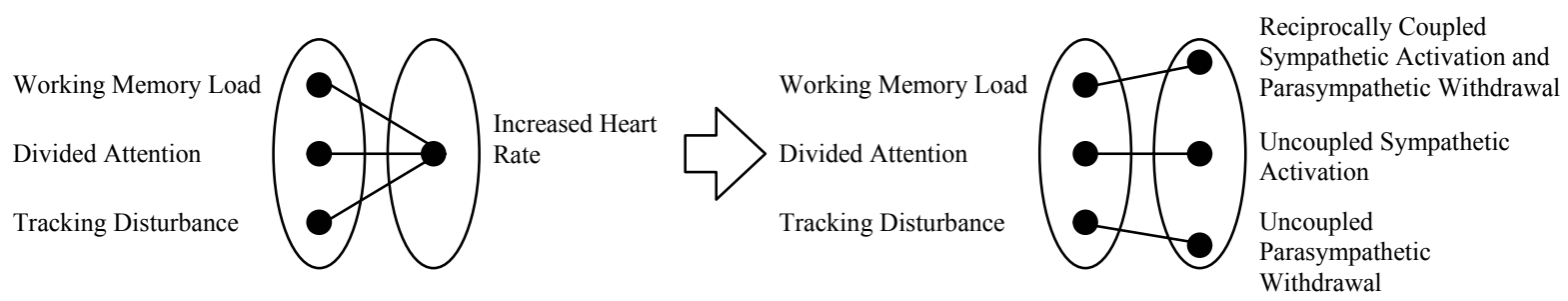

Figure 1. Many-to-one (left panel) and one-to-one (right panel) psychological-physiological mappings

Because past research has provided information about the psychological processes involved with the three tasks, we can make tentative conclusions about the psychological-physiological mappings. In particular, the results of Backs (1995) showed that uncoupled sympathetic activation is elicited by central processing, whereas uncoupled parasympathetic withdrawal is elicited by perceptual/motor processing. Reciprocally coupled sympathetic activation and parasympathetic withdrawal is elicited by a combination of the two psychological processes (central processing and perceptual/motor processing). However, while these results are promising, they must remain tentative until more confirmatory evidence of psychologicalphysiological mappings is established.

\section{DECIPHERING PSYCHOLOGICAL-PHYSIOLOGICAL MAPPINGS WHILE DRIVING}

Recent research has just begun to look at different facets of the driving task and the different modes of autonomic control elicited by them (Backs, Lenneman, Wetzel, and Green, in press). In this study, driving a simulated road with curves of varying degrees was shown to elicit similar changes in heart rate, but different modes of autonomic control. In particular, three curves with radii of 582, 291, and 194 meters elicited faster heart rates. However, while the 582- and 194meter curves elicited uncoupled parasympathetic withdrawal, the 291-meter curve elicited reciprocally coupled sympathetic activation and parasympathetic withdrawal. Past research has shown that uncoupled parasympathetic withdrawal is elicited by perceptual/motor processing, while sympathetic activation is elicited by central processing (Backs, 1995; Lenneman and Backs, 2000). The results indicated that driving through 582- and 194-meter curves elicits more perceptual/motor processing, whereas driving through a 294-meter curve elicits central processing in addition to perceptual/motor processing (see Figure 2). 


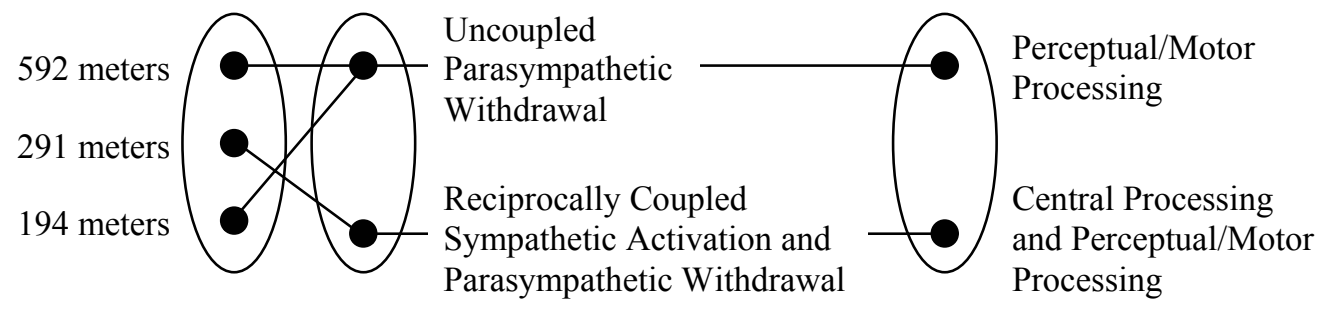

Figure 2. Mapping between curve radius, autonomic control modes, and psychological processes

Assuming that the 582- and 194-meter curves were the easiest and hardest curves to drive, respectively, it then seems counterintuitive that they would elicit the same autonomic control mode while the 291-meter curve elicited a different autonomic control mode. As the radius of the curves decreased, increased sympathetic activation coupled with parasympathetic withdrawal should be expected. But the sympathetic activation that was elicited by the 291-meter curve was not elicited by the 194-meter curve. This discrepancy in measurements of autonomic control can be understood when one considers the differences in difficulty of driving the three curves.

Undoubtedly, the 582-meter curve was the easiest to drive. It is likely that no higher-order strategy was needed to maintain position on the road. Since few central processing resources were needed, the 582-meter curve elicited uncoupled parasympathetic withdrawal. In contrast, the 194-meter curve was the hardest curve to drive and we believe that a higher-order strategy was needed to maintain position on the road. However, we believe that driving through this curve was so difficult that the perceptual/motor processing resources needed to maintain position left few resources to perform the calculations required for a higher-order strategy to maintain position. Thus, because few resources were available to perform any central processing strategy, the 194-meter curve also elicited uncoupled parasympathetic withdrawal.

In order to account for the autonomic control mode elicited by the 291-meter curve, consider that the 291-meter curve is likely not as easy as the 582-meter curve or as difficult as the 194-meter curve. For the 291-meter curve, we believe it is possible that the subjects were able to perform the perceptual/motor processing necessary and also use some form of central processing strategy to help maintain position. Thus, the 291-meter curve elicited reciprocally coupled sympathetic activation and parasympathetic withdrawal.

This explanation is supported by the heart rate data. Although the data shows an increase in heart rate for all three curves, heart rate was actually slower in the 194-meter curve than the 582- and 291-meter curves. This result was also observed in a field study by Richter et al. (1998). They observed increases in heart rate as the curves ranged from high to moderate widths of curve radius, but then observed decreases in heart rate as the curve reached its smallest radius. Lacey (1969) was able to show that heart rate actually decreased during the intake of large amounts of perceptual information. These results support the theory that the perceptual/motor demands of the 194-meter curve were considerably higher than the other curves, removing any possibility of performing some strategy to negotiate the curve. 


\section{CONCLUSION}

The efficacy of the autonomic space approach to the diagnosis of psychological processes involved in a small facet of the driving task has been demonstrated. More research needs to be done to build upon the understanding of the relation between the physiological and psychological domains. This includes exploring higher order psychological functions and their relations to autonomic space across a wide variety of information processing tasks. Previous research has begun to explore these relationships in the lab (Lenneman and Backs, 2000). In addition, higher order physiological functions (e.g., assessments across multiple visceral organs or as the physiological measures change across time) should be explored to try and develop the highest possible level of diagnosticity.

\section{REFERENCES}

Backs, R.W. (1995). Going beyond heart rate: Modes of autonomic control in the cardiovascular assessment of mental workload. The International Journal of Aviation Psychology, 5, 25-48.

Backs, R.W. (2001). An autonomic space approach to the psychophysiological assessment of mental workload. In P.A. Hancock, \& P.A. Desmond (Eds.), Stress, Workload, and Fatigue (pp. 279-289). Mahwah, NJ: Lawrence Erlbaum Associates.

Backs, R.W., Lenneman, J.K., \& Sicard, J.L. (1999). The use of autonomic components to improve cardiovascular assessment of mental workload in flight simulation. The International Journal of Aviation Psychology, 9, 33-47.

Backs, R.W., Lenneman, J.K., Wetzel, J.M., \& Green, P. (in press). Cardiac measures of driver workload during simulated driving with and without visual occlusion. Human Factors.

Berntson, G.G., Cacioppo, J.T., \& Quigley, K.S. (1991). Autonomic determinism: The modes of autonomic control, the doctrine of autonomic space, and the laws of autonomic constraint. Psychological Review, 98, 459-487.

Cacioppo, J.T., \& Tassinary, L.G. (1990). Inferring psychological significance from physiological signals. American Psychologist, 45, 16-28.

Cannon, W.B. (1939). The Wisdom of the Body. New York: Norton.

Lacey, J.I. (1969). Autonomic indices of attention, readiness, and rejection of the external environment. In D.P. Kimble (Ed.), Readiness to remember. New York: Gordon and Breach.

Lenneman, J.K., \& Backs, R.W. (2000). The validity of factor analytically derived autonomic components for mental workload assessment. In R.W. Backs, \& W. Boucsein (Eds.), Engineering Psychophysiology: Issues and Applications (pp. 161-175). Mahwah, NJ: Lawrence Erlbaum Associates.

Lewis, C.E., Jr., Jones, W.L., Austin, F., \& Roman, J. (1967). Flight research program: IX. Medical monitoring of carrier pilots in combat-II. Aerospace Medicine, 38, 581-592.

O’Donnell, R.D., \& Eggemeier, F.T. (1986). Workload assessment methodology. In K.R. Boff, L. Kaufman, \& J.P. Thomas (Eds.), Handbook of perception and human performance: Vol. II. Cognitive processes and performance (pp. 42-1-42-49). New York: Wiley Interscience.

Roscoe, A.H. (1978). Stress and workload in pilots. Aviation, Space, and Environmental Medicine, 49, 630-636. 